\title{
Refreshing the strategy
}

\author{
We reflect on some aspects of the newly updated plan of the US National Nanotechnology Initiative.
}

On 31 October the National Nanotechnology Initiative (NNI) published its 2016 Strategic Plan (www.nano.gov/node/1676). The document updates the previous version of the strategic plan from February 2014, and intends to highlight the priorities that all the federal agencies participating in the NNI agreed upon for the next three years. It is perhaps fair to say that the document does not contain big surprises, and that the main updates had already been announced during the past couple of years. We feel, however, that it is worth reflecting on some aspects that emphasize the way in which the US funding bodies intend to approach nanotechnology in the next few years.

The NNI remains founded on four main objectives that can be summarized as the advance of research and development, the commercialization of new nanotechnologies, the fostering of nanotechnology education and the support for the responsible development of nanotechnology. It is clear however that commercialization is high on the
NNI agenda. The document spotlights (1) an initiative launched in October 2015 to advance the commercialization of nanotechnology inventions related to cancer, (2) a public-private partnership for the realization of technology to monitor human performance, and (3) the Department of Agriculture's efforts to commercialize cellulosic nanomaterials for a range of applications.

Also notable is the evolution of the Nanotechnology Signature Initiatives (NSIs). The launch in March this year of the NSI for Water Sustainability marked a clear aspiration to tackle a global issue, which is becoming more and more pressing for the US itself. Conversely, it is interesting to learn that the NSI for Solar Energy Collection and Conversion was retired, primarily because "a robust research ecosystem has been established to support nanotechnology-based solar energy R\&D." In a way this can be seen as a testimony of the transient nature of the NNI, whose goal seems to create the foundations for research and commercialization of nanotechnology.

Arguably the most obvious update from the 2014 version of the strategy plan is the introduction of the nanotechnologyinspired grand challenges and the following launch of the first one in October 2015 the realization of a new type of computer. Although assessing their efficacy will require time, the concept of the grand challenges, which should interest public and private funding alike, is certainly intriguing. Unfortunately, the concept was also strictly related to President Obama's approach to scientific and technological development. Given the uncertainty at this stage regarding Donald Trump's approach to science, it would have been desirable to see a second announcement before election day on 8 November. Now we'll probably have to wait till the first review of the programme in October 2017 to learn something about the future of the grand challenges, and perhaps of the US nanotechnology programme altogether.

\section{Analyse that}

\section{With the publication of the first Nature Nanotechnology Insight, we also introduce the concept of Analysis as an article type to our journal.}

\begin{abstract}
The goal of Nature Nanotechnology
has always been to publish the most significant research advances in all areas of nanoscience and nanotechnology. In a manuscript, we look for fundamental advance or strong indication of potential for technological development. We have no intention of changing this goal in the future. We are also aware, however, that the use of nanomaterials for applications that address societal challenges requires studies that address issues like efficiency, cost and potential damage to the environment or human health and we feel that we should increase coverage of such studies in our journal.

In the Insight included in this issue we publish or first Analysis. This article type has been used by some of our sister journals including Nature, Nature Genetics,
\end{abstract}

Nature Biotechnology and more recently Nature Energy. The main purpose of such articles is to provide new analyses of existing data to obtain insights that would help formulate strategies for the solution of large-scale problems. The Analysis by Linda Ager-Wick Ellingsen and co-authors (page 1039) provides little new scientific insight into the properties of nanomaterials. Rather, by digging out data from existing literature, they screen a range of nanomaterials currently used for batteries and fuel-cell applications and carry out an early-stage life-cycle analysis in an attempt to identify the nanomaterials that can best contribute to $\mathrm{CO}_{2}$ emission reduction.

We should clarify that because the Analysis by Ellingsen and co-authors is part of a special issue, we allowed it a special format. Thus it includes a box and the main text is very long. Our model format for future Analyses is that of research Articles, though we'll evaluate the possibility of allowing longer text on a case-by-case basis. Most importantly, like Articles, Analyses will be properly peer reviewed. They will also be subject to selective assessment criteria and evaluation for their editorial suitability for our journal: the results and conclusions should be important enough to be appreciated by a wide audience including non-experts in the specific field reported.

In the future it will be possible to select Analysis as the manuscript type during the submission process. In the meantime, we shall evaluate after submission whether a manuscript should be considered an Analysis, but feel free to indicate this as your preference in your cover letter. 